\title{
Evaluación del contenido de metales pesados en las aguas del Río Yamanigüey
}

\author{
Evaluation of the content of heavy metals in the waters of the Yamanigüey \\ River
}

\author{
Pedro Luis Dunán Avila ${ }^{1, a}$, Moraima Fernández Rodríguez ${ }^{1, b}$, Amalia Beatriz Riverón Zaldívar ${ }^{1, c}$, Pedro \\ Rafael Bassas Noa ${ }^{2, d}$
}

Recibido: 16/01/2021 - Aprobado: 25/10/2021 - Publicado: 23/12/2021

\begin{abstract}
RESUMEN
Los metales pesados se encuentran entre los contaminantes ambientales más tóxicos de los últimos tiempos. La presente investigación tuvo como objetivo evaluar las aguas del Río Yamanigüey según normas nacionales e internacionales para su uso como agua potable, por ser este unos de los ríos principales de la región de Moa. Se recolectaron 12 muestras a lo largo de toda la cuenca para evaluar los contenidos de aluminio (Al), cromo $(\mathrm{Cr})$, cromo hexavalente $(\mathrm{Cr}+6)$, manganeso $(\mathrm{Mn})$, hierro $(\mathrm{Fe})$, cobalto $(\mathrm{Co})$, níquel $(\mathrm{Ni})$, cobre $(\mathrm{Cu})$, cinc $(\mathrm{Zn})$ y plomo $(\mathrm{Pb})$ mediante el método de espectrofotometría de absorción atómica. Los resultados fueron contrastados con las normas correspondientes de la "Guías para la calidad del agua de consumo humano", con la Norma Cubana (NC 1021, 2014) "Higiene comunal - fuentes de abastecimiento de agua - calidad y protección sanitaria y la Norma Cubana (NC 827, 2017) "Agua potable - requisitos sanitarios". Como principales resultados se obtuvo que el cromo, el manganeso, el cobre y el zinc, en el $100 \%$ de las muestras estudiadas del Río Yamanigüey, según las normas evaluadas se localizan por debajo de los límites máximos admisibles (LMA).
\end{abstract}

Palabras claves: Agua potable; metales pesados; normas; río; Yamanigüey.

\begin{abstract}
Heavy metals are among the most toxic environmental pollutants in recent times. The objective of this research was to evaluate the waters of the Yamanigüey River according to national and international standards for its use as drinking water, as this is one of the main rivers in the Moa region. Twelve samples were collected throughout the entire basin to evaluate the contents of aluminum (Al), chromium ( $\mathrm{Cr}$ ), hexavalent chromium (Cr+6), manganese $(\mathrm{Mn})$, iron ( $\mathrm{Fe})$, cobalt (Co), nickel (Ni), copper $(\mathrm{Cu})$, zinc $(\mathrm{Zn})$ and lead $(\mathrm{Pb})$ using the atomic absorption spectrophotometry method. The results were contrasted with the corresponding norms of the WHO (2011) "Guidelines for the quality of water for human consumption", with the Cuban Norm (2014) "Communal hygiene - sources of water supply - quality and sanitary protection and the Cuban Norm (2017) "Drinking water - sanitary requirements". The main results were that chromium, manganese, copper and zinc, in $100 \%$ of the samples studied from the Yamanigüey River, according to the standards evaluated, are located below the maximum admissible limits (MAL).
\end{abstract}

Keywords: Drinking water; heavy metals; standards; river; Yamanigüey.

\footnotetext{
1 Universidad de Moa “Dr. Antonio Núñez Jiménez”, Facultad de Geología y Minería. Avenida Calixto García Iñiguez \#15 entre Avenida 7 de Diciembre y Calle Reynaldo Laffita Rueda, Reparto Caribe, Moa, Holguín, Cuba.

2 Centro de Investigaciones del Níquel "Capitán Alberto Fernández Montes de Oca". Carretera Yagrumaje Km. 7, Moa, Holguín, Cuba.

a Autor para correspondencia: pdavila@ismm.edu.cu - ORCID: http://orcid.org/0000-0002-0022-6662

E-mail: mfernandez@ismm.edu.cu - ORCID: http://orcid.org/0000-0003-1211-3865

E-mail: briveron@ismm.edu.cu - ORCID: http://orcid.org/0000-0001-7155-5626

d E-mail: pbassas@cil.moa.minem.cu - ORCID: http://orcid.org/0000-0002-6690-2539
} 


\section{INTRODUCCIÓN}

La calidad del agua potable, su disponibilidad y acceso, son un derecho humano en la política nacional cubana. Con el decursar del tiempo, las autoridades competentes se han percatado de la necesidad de la búsqueda y obtención de agua potable segura, para garantizar la prevención de enfermedades asociadas al consumo de este recurso con una calidad inadecuada. Según la (OMS, 2011a), el agua potable es aquella que no ocasiona ningún riesgo significativo a la salud de las personas de diferentes edades cuando se consume por un largo periodo de tiempo. Éstas, pierden su potabilidad, cuando sus propiedades fisicoquímicas y bacteriológicas se encuentran fuera de los rangos permisibles por los estándares de calidad en vigor para agua potable. La calidad del agua de los ríos se deteriora debido a que a ellos llegan los desechos sólidos y líquidos generados por las viviendas, las industrias, la agricultura y la acción de las aguas superficiales de escorrentías. Día tras día, el consumo del recurso agua aumenta y por consiguiente las fuentes de suministro se encuentran amenazadas por las contaminaciones que genera el hombre. Es por lo que, se hace necesario, realizar controles sistemáticos del recurso mediante el monitoreado de su calidad. Para conocer la calidad del agua de un río es de vital importancia determinar el grado o nivel de contaminación que posee y la capacidad que presenta éste de recuperarse a las mismas características o condiciones similares a las que poseía antes de haber sido afectado por los agentes contaminantes.

Existen varios contaminantes que afectan la calidad de los cuerpos de agua, pero los metales pesados se destacan entre los más perjudiciales, puesto que no se degradan, sino que permanecen en él por largos periodo de tiempo. Con la realización de investigaciones científicas, se ha comprobado que estos metales al presentar valores fuera de normas ocasionan daños morfológicos y fisiológicos en los organismos (García-García, N., Pedraza-Garciga, J., Montalvo, J. F., Martínez, M., \& Leyva, 2012); (White \& Rasmussen, 1998). Por su alto potencial tóxico el $\mathrm{Cr}, \mathrm{Cr}^{+6}$, el $\mathrm{Pb}$, etc, son objeto de atención en todas las investigaciones de calidad de agua para el consumo, por estar considerados como metales altamente peligrosos; sin embargo, otro grupo de metales entre los que se encuentran el $\mathrm{Cu}$ y el $\mathrm{Zn}$ pueden resultar tóxicos si su concentración excede los niveles permitidos por las normas (GarcíaGarcía, N., Pedraza-Garciga, J., Montalvo, J. F., Martínez, M., \& Leyva, 2012).

Debido al crecimiento poblacional e industrial en los últimos años, se ha hecho necesario la búsqueda de agua con las características indispensables para agua potable. Son cuantiosos los trabajos que se han realizado en Cuba y el Mundo sobre esta temática en los últimos años (Lima Cazorla et al., 2005); (Fernández Rodríguez, Moraima; Fernández Urgellés, 2007); (Rivera et al., 2013); (Chávez-Vallarino, 2011); (Argota-Pérez \& Argota-Coello, 2012); (Salas Urviola, 2014); (Espitia, 2015); (Flores Lozano, 2016); (Vidal, N. F., Acosta, H. S., \& Villacres, 2018); (Lopez Arisaca, 2018); (Arada Pérez et al., 2018); (Fernández-Rodríguez et al., 2018); (Gonzáles V, Valle Sonia, Nirchio M., Olivero J., Tejeda L, Valdelamar J, 2018). En Yamanigüey (lugar donde se encuentra el río objeto de estudio), se han realizado investigaciones con vista a estudiar morfotectónicamente la zona (DomínguezGonzález, L., Rodríguez-Infante, A., Wobbe, F., Stanek, K. P., \& Gloaguen, 2007); (Barea Pérez et al., 2010), y con el objetivo de evaluar la calidad del agua mediante los índices de calidad de las aguas (ICA) en el asentamiento poblacional y en el río (Crespo-Lambert, 2018); (Dunán Ávila, 2019), pero en estos no se ha estudiado los metales pesados con suficiente detalle, es por lo que, se hace necesario la evaluación de los metales pesados en las aguas del Río Yamanigüey según normas nacionales e internacionales para agua potable.

\section{MÉTODOS}

\subsection{Localización del área de estudio}

El área de estudio se localiza en Yamanigüey, poblado perteneciente al municipio Moa, de la provincia Holguín, Cuba (Figura 1). El río objeto de estudio es de gran importancia para el municipio, por ser unas de las mayores reservas de aguas superficiales existentes y de la cual se alimenta gran parte del poblado Yamanigüey. Las precipitaciones (periodo 2010-2019) oscilan entre 1469,9 - 3014,4 mm/año, por lo que se considera unas de las regiones más lluviosas del país. Para el estudio de las precipitaciones existentes en el período se utilizó el pluviómetro localizado en el poblado de Cañete, Moa, Holguín, Cuba, en las coordenadas X: 716,70 Y: 213,40 enclavado a una altura de $200 \mathrm{msnm}$. El territorio de Moa presenta una elevada complejidad desde el punto de vista orográfico, apreciándose un predominio en el relieve de montaña en la parte este con cotas son superiores a los 450 metros y valores máximos de isobasitas de 900 metros en el segundo orden y 800 metros en el tercero y ondulado hacia la parte norte del municipio (Polanco-Almaguer, 2013).

\subsection{Muestreo y análisis fisicoquímico del agua}

Para la presente investigación se recolectaron 12 muestras de agua en el Río Yamanigüey en periodo de seca (noviembre), para estudiar las propiedades fisicoquímicas de ella. Estos parámetros se estudiaron siguiendo la norma (APHA, 2016) y se ubicaron en un mapa digital mediante la utilización del programa QGIS 3.9.0 (Figura 1). Las concentraciones de $\mathrm{Al}, \mathrm{Cr}, \mathrm{Cr}^{+6}, \mathrm{Mn}, \mathrm{Fe}, \mathrm{Co}, \mathrm{Ni}$, $\mathrm{Cu}, \mathrm{Zn}$ y $\mathrm{Pb}$ se determinaron mediante el método de espectrofotometría de absorción atómica utilizando el espectrofotómetro modelo ATI Unicam. Los análisis fisicoquímicos se realizaron por duplicado, para garantizar una mejor confiabilidad de los resultados, en la Unidad de Proyectos de Laboratorio del Centro de Investigaciones del Níquel, Moa, Holguín, Cuba; la cual cuenta con 14 ensayos acreditados por la norma cubana NC: ISO/IEC-17025 del 2006 (Requisitos generales para la competencia técnica de los laboratorios de ensayos y calibración), para el análisis de agua y aguas residuales. El análisis y procesamiento estadístico de datos se realizó mediante la herramienta Microsoft Excel. Para una mejor claridad, comprensión y organización de los resultados se separó el estudio en tres grupos de metales en relación con el número atómico que presentan cada uno de ellos, comenzando por los metales que tienen menor número atómico hasta los de mayor número atómico. 


\section{RESULTADOS Y DISCUSIÓN}

3.1. Evaluación del aluminio, cromo, cromo hexavalente y manganeso en las aguas del Río Yamanigüey

La siguiente tabla muestra la concentración de aluminio, cromo, cromo hexavalente y manganeso en las aguas del Río Yamanigüey y su correspondiente valor normado por los estándares nacionales e internacionales para agua potable.

\begin{tabular}{ccccc}
\hline \multirow{2}{*}{ Muestra } & \multicolumn{4}{c}{ Metales (mg/L) } \\
& Al & Cr & Cr $^{+6}$ & Mn \\
\hline Y-1 & 0,11 & 0,01 & 0,00 & 0,02 \\
Y-2 & 0,01 & 0,00 & 0,00 & 0,00 \\
Y-3 & 0,05 & 0,01 & 0,00 & 0,01 \\
Y-4 & 0,05 & 0,00 & 0,00 & 0,00 \\
Y-5 & 0,00 & 0,00 & 0,00 & 0,00 \\
Y-6 & 0,04 & 0,01 & 0,00 & 0,02 \\
Y-7 & 0,20 & 0,00 & 0,00 & 0,02 \\
Y-8 & 0,00 & 0,00 & 0,00 & 0,00 \\
Y-9 & 0,00 & 0,00 & 0,00 & 0,01 \\
Y-10 & 0,02 & 0,00 & 0,00 & 0,00 \\
Y-11 & 0,01 & 0,00 & 0,00 & 0,00 \\
Y-12 & 0,03 & 0,00 & 0,00 & 0,00 \\
NC (2014) & NR & 0,05 & NR & 0,10 \\
NC (2017) & 0,20 & 0,05 & NR & 0,10 \\
OMS (2011) & NR & 0,05 & NR & NR \\
\hline
\end{tabular}

NR: No reportado en la norma.
El aluminio, según la norma de la (OMS, 2011b) es el elemento metálico más abundante de la corteza terrestre constituyendo el $8 \%$ aproximadamente de su composición total. Los valores del metal en las aguas del Río Yamanigüey oscilan entre $0.00-0.20 \mathrm{mg} / \mathrm{L}$. Tomando como referencia la Norma Cubana (NC 827, 2017), la muestra Y-7 se encuentra en el límite máximo admisible y las 11 muestras restantes se localizan por debajo del valor de referencia reglamentado en la norma $(0.2 \mathrm{mg} / \mathrm{L})$. La concentración de aluminio en aguas naturales (lagunas, lagos, arroyos) generalmente es menor de $0.1 \mathrm{mg} / \mathrm{L}$ según la (OMS, 2011a) pero en las aguas del Río Yamanigüey encontramos valores hasta de $0.2 \mathrm{mg} / \mathrm{L}$. Como promedio el aluminio en las aguas del Río Yamanigüey es de $0.04 \mathrm{mg} / \mathrm{L}$ (resultado inferior a los tomados como referencia en las normas analizadas durante la investigación).

El cromo se encuentra distribuido ampliamente en la capa más superficial del planeta Tierra con valencias que van desde +2 hasta +6 . Generalmente, los contenidos de cromo en las aguas de los ríos son bajos, a menos que estos cuerpos de agua atraviesen rocas compuestas por el elemento químico (OMS, 2011a). La Norma Cubana (NC 827, 2017), (NC 1021, 2014) y la norma de la (OMS, 2011a) presentan como valor máximo admisible en las muestras de agua potable $0.05 \mathrm{mg} / \mathrm{L}$, encontrándose todas las muestras de agua analizadas con resultados inferiores a los normados. El cromo oscila entre $0.00-0.01 \mathrm{mg} / \mathrm{L}$ en las muestras objeto de estudio. El valor promedio del contenido del cromo en las muestras de agua del río es de $0.01 \mathrm{mg} / \mathrm{L}$, lo que cumple con las normas evaluadas para que sus aguas sean potables.
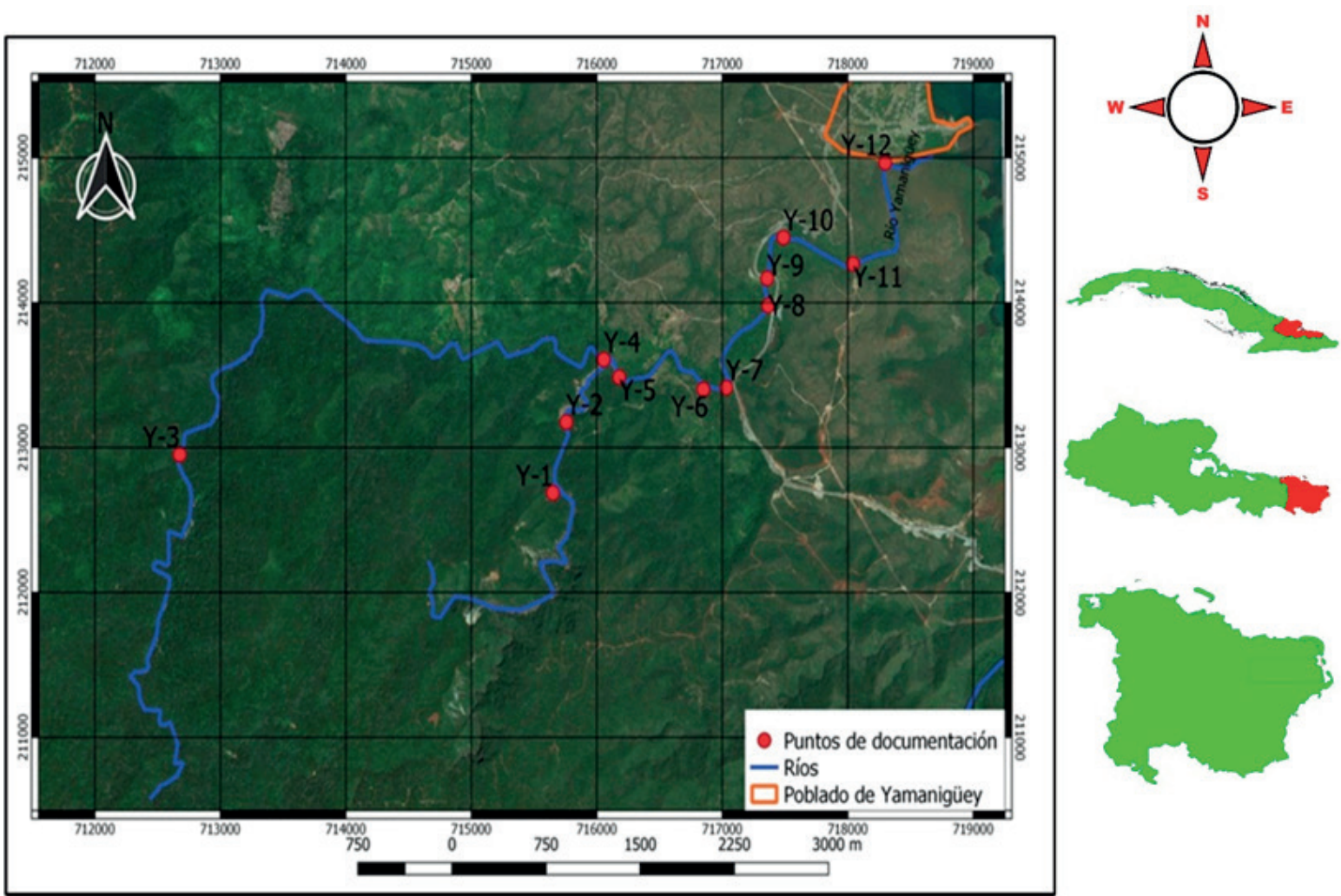

Figura 1. Representación gráfica del mapa de ubicación geográfica y los puntos de muestreo. 
El cromo hexavalente en las 12 muestras estudiadas es de $0.00 \mathrm{mg} / \mathrm{L}$. Este elemento químico es un peligro potencial en la salud de los humanos por ser altamente tóxico, lo que posibilita la alteración del material genético del organismo y causar cáncer rápidamente. Las normas evaluadas no recogen ningún valor de referencia para este elemento químico, pero en las aguas de consumo humano la concentración del metal debe lo más bajo posible. Es de importante enunciar que las aguas del Río Yamanigüey corren por encima de cuerpos cromíferos en la parte alta de la cuenca, pero en la etapa de laboratorio realizada durante la investigación no se encontraron valores de cromo elevados, es por lo que, debe ser un tema para considerar para próximos estudios.

El manganeso es un elemento abundante en la corteza terrestre y lo podemos localizar junto al hierro y otros metales. Según la norma de la (OMS, 2011a), al metal, lo podemos encontrar en fuentes de agua tanto superficial como subterráneas de manera natural, específicamente cuando hay presencia de microoxidación. Para la Norma Cubana (NC 827, 2017) y (NC 1021, 2014) el LMA es de $0.1 \mathrm{mg} / \mathrm{L}$, lo que cumplen con esto las 12 muestras de agua objeto de estudio. Según investigaciones realizados la concentración del manganeso, es por lo general, menor que la del hierro, cumpliéndose en las aguas del Río Yamanigüey. Como promedio los niveles de manganeso encontrado en las aguas del río son de $0.01 \mathrm{mg} / \mathrm{L}$. El metal objeto de estudio en las aguas del río oscila entre 0,00 $\mathrm{mg} / \mathrm{L}-0,02 \mathrm{mg} / \mathrm{L}$, lo que indica un mínimo y un máximo respectivamente. El manganeso, combinado con el calcio, zinc y cobre, constituyen elementos de vital importancia para el buen estado y funcionamiento de los huesos existentes en los seres humanos cuando se encuentran en las concentraciones normadas, porque en contenidos que excedan los valores estandarizados puede ser perjudicial para la salud humana. Debido a los bajos contenidos de manganeso en las aguas del Río Yamanigüey, este no representa un riesgo para la salud de los pobladores.

\subsection{Evaluación del hierro, cobalto, níquel y cobre en las aguas del Río Yamanigüey}

La siguiente tabla 1 muestra la concentración de hierro, cobalto, níquel y cobre en las aguas del Río Yamanigüey y su correspondiente valor normado por los estándares nacionales e internacionales para agua potable.

El hierro es un metal que lo encontramos constantemente en nuestro alrededor, ya sea en las rocas por donde transitamos o en construcciones. Los contenidos de hierro en las aguas del Río Yamanigüey oscilan entre 0.01- $0.34 \mathrm{mg} / \mathrm{L}$. Las normas (NC 827, 2017), (NC 1021, 2014) y (OMS, 2011a) recogen como valor de referencia para el hierro $0.3 \mathrm{mg} / \mathrm{L}$, donde las muestras $\mathrm{Y}-11$ y $\mathrm{Y}-12$ se encuentran por encima de este valor. El alto contenido de hierro en estos puntos se debe a la presencia de metales dentro del cauce del río en proceso de oxidación, además de la litología por donde corren sus aguas (rocas ultramáficas serpentinizadas y corteza de meteorización en la parte baja del valle del río). Para el hierro, el promedio es de 0.08 $\mathrm{mg} / \mathrm{L}$, valor que se localiza por debajo de los LMA por las normas evaluadas.

El cobalto, podemos encontrarlo de forma natural, en las rocas compuestas por el metal, lo que posibilita la formación de depósitos minerales; en el suelo, producto de la descomposición de las rocas, a la existencia de suelos contaminados por el tráfico de carreteras y de aeropuertos. Se puede localizar cobalto en el aire a raíz del proceso metalúrgico, por el uso de incineradores que utilizan carbón como combustible y a causa del tubo de escape de vehículos. El metal cobalto lo encontramos en el agua por

Tabla 1. Concentración de hierro, cobalto, níquel y cobre en las aguas del Río Yamanigüey

\begin{tabular}{ccccc}
\hline \multirow{2}{*}{ Muestra } & \multicolumn{4}{c}{ Metales (mg/L) } \\
& $\mathrm{Fe}$ & $\mathrm{Co}$ & $\mathrm{Ni}$ & $\mathrm{Cu}$ \\
\hline Y-1 & 0,01 & 0,02 & 0,04 & 0,01 \\
Y-2 & 0,03 & 0,01 & 0,15 & 0,01 \\
Y-3 & 0,13 & 0,02 & 0,06 & 0,01 \\
Y-4 & 0,04 & 0,01 & 0,00 & 0,00 \\
Y-5 & 0,01 & 0,02 & 0,02 & 0,00 \\
Y-6 & 0,03 & 0,02 & 0,07 & 0,00 \\
Y-7 & 0,03 & 0,02 & 0,03 & 0,01 \\
Y-8 & 0,00 & 0,00 & 0,00 & 0,00 \\
Y-9 & 0,00 & 0,02 & 0,00 & 0,01 \\
Y-10 & 0,04 & 0,03 & 0,00 & 0,01 \\
Y-11 & 0,33 & 0,00 & 0,30 & 0,01 \\
Y-12 & 0,34 & 0,00 & 0,30 & 0,01 \\
NC (2014) & 0,30 & NR & 0,02 & 2,00 \\
NC (2017) & 0,30 & NR & 0,02 & 2,00 \\
OMS (2011) & 0,30 & NR & 0,07 & 2,00 \\
\hline
\end{tabular}

NR: No reportado en la norma. 
la existencia de rocas en el cauce de los ríos compuestas por el metal, lo que posibilita que el agua, con su poder disolvente, incorpore a su composición química estos elementos que encuentra a su paso. También el cobalto puede incorporase a las aguas superficiales a raíz de la escorrentía producida por la lluvia que cae en suelos y rocas que presentan alto contenido del elemento químico objeto de estudio (ATSDR, 2000). El cobalto en las aguas del Río Yamanigüey oscila entre $0.00-0.03 \mathrm{mg} / \mathrm{L}$ y el promedio del metal en las aguas del río es de $0.01 \mathrm{mg} / \mathrm{L}$. Las normas utilizadas no recogen ningún valor de referencia para el elemento químico estudiado.

Los valores de níquel en las aguas del río objeto de estudio oscilan entre $0.00-0.30 \mathrm{mg} / \mathrm{L}$. Al analizar las muestras Y-1, Y-2, Y-3, Y-6, Y-7, Y-11, Y-12 por la Norma Cubana (NC 827, 2017) y (NC 1021, 2014), los valores de las muestras se encuentran por encima de los límites máximos admisibles $(0.02 \mathrm{mg} / \mathrm{L}), \mathrm{Y}-5$ se localiza en el límite máximo, y las muestras Y-4, Y-8, Y-9, Y-10 se están por debajo del límite máximo admisible. Con respecto a la norma de la (OMS, 2011a) las muestras Y-1, Y-3, Y-4, Y-5, Y-7, Y-8, Y-9, Y-10 se localizan por debajo del LMA por ella $(0.07 \mathrm{mg} / \mathrm{L})$, las muestras Y-2, Y-11, Y-12 se encuentran por encima de la norma y la muestra Y-6 se encuentra en el límite máximo admisible por la norma. Como promedio, el níquel en las aguas del Río Yamanigüey es de $0.08 \mathrm{mg} / \mathrm{L}$, encontrándose por encima de límite máximo admisible (LMA) por las normas evaluadas, debiéndose esto a la presencia de cortezas de meteorización ferroniquelíferas de pequeña potencia en la parte baja del valle del río, además de las litologías por donde corren sus aguas que son ultramáficas serpentinizadas.

La (OMS, 2011a) plantea que, por lo general, la existencia de cobre en las aguas para el consumo humano procede de la corrosión en las tuberías cuyo material de fabricación es el cobre. También, podemos encontrar cobre en las aguas superficiales a causa de la contaminación de suelos con este metal o mineralización cuprífera existente cerca del cauce de los ríos. Éste, es un nutriente esencial para la salud de los seres humanos y, puede ser considerado un agente contaminante del agua de consumo si los niveles de este exceden los límites admisibles por las normas vigentes. Las normas (OMS, 2011a), (NC 827, 2017) y (NC 1021, 2014) plantean como valor de referencia para el cobre en las aguas de $2.0 \mathrm{mg} / \mathrm{L}$, lo que cumplen con esto las 12 muestras evaluadas. Como promedio la concentración de cobre es de $0.01 \mathrm{mg} / \mathrm{L}$, lo que cumple con los LMA de las normas evaluadas. Las concentraciones de cobre en las aguas del río se localizan entre $0,00 \mathrm{mg} / \mathrm{L}-0,01 \mathrm{mg} / \mathrm{L}$, lo que indica un mínimo y un máximo respectivamente. Debido a la baja concentración del cobre existente en las aguas del Río Yamanigüey, este no presenta riesgos para la salud.

\subsection{Evaluación del zinc y plomo en las aguas del Río Yamanigüey}

La siguiente tabla 2 la concentración de zinc y plomo en las aguas del Río Yamanigüey y su correspondiente valor normado por los estándares nacionales e internacionales para agua potable.
Tabla 2. Concentración de zinc y plomo en las aguas del Río Yamanigüey

\begin{tabular}{lcc}
\hline Muestra & \multicolumn{2}{c}{ Metales $(\mathrm{mg} / \mathrm{L})$} \\
\hline Y-1 & Zn & $\mathrm{Pb}$ \\
Y-2 & 0,01 & 0,03 \\
Y-3 & 0,02 & 0,03 \\
Y-4 & 0,01 & 0,02 \\
Y-5 & 0,03 & 0,00 \\
Y-6 & 0,03 & 0,00 \\
Y-7 & 0,02 & 0,04 \\
Y-8 & 0,02 & 0,03 \\
Y-9 & 0,01 & 0,00 \\
Y-10 & 0,02 & 0,04 \\
Y-11 & 0,02 & 0,00 \\
Y-12 & 0,04 & 0,00 \\
NC (2014) & 0,02 & 0,00 \\
NC (2017) & 5,00 & 0,05 \\
OMS (2011) & 5,00 & 0,05 \\
\hline
\end{tabular}

NR: No reportado en la norma.

El zinc es un oligoelemento de vital importancia para los seres humanos y se encuentra en el agua potable principalmente como sales o complejos orgánicos, lo que indica que la principal fuente de zinc en el cuerpo humano son los alimentos (Rubio et al., 2007). Según la (OMS, 2011a) los niveles en que halla el zinc en los distintos cuerpos de agua utilizados como potable no son un problema para la salud. El valor promedio del zinc en las aguas del río es de $0.01 \mathrm{mg} / \mathrm{L}$. La Norma Cubana (NC 827, 2017) y (NC 1021, 2014) recogen como valor máximo admisible en las aguas de consumo $5.0 \mathrm{mg} / \mathrm{L}$, lo que cumplen con esto las 12 muestras evaluadas.

Según la norma (OMS, 2011a) el plomo se incorpora en las aguas potables y en los ríos como resultado de la disolución de fuentes naturales con gran contenido de plomo, es decir, por la presencia de minerales como la galena, cerusita, anglesita, entre otros. También su presencia en el agua de consumo está asociada la existencia accesorios y aleaciones que usan el plomo en las tuberías utilizadas para el transporte del agua. El valor de referencia $(0.01 \mathrm{mg} / \mathrm{L})$ es provisional y se escoge sobre la base de la efectividad del tratamiento que se le realice en las plantas potabilizadoras y la capacidad de las técnicas analíticas que se utilicen de detectar el elemento químico, es por lo que las concentraciones deben ser tan bajas como sea posible. Las muestras de agua Y-1, Y-2, Y-3, Y-6, Y-7, Y-9, se encuentran por encima del límite máximo admisible por las normas evaluadas. Las muestras Y-4, Y-5, Y-8, Y-10, Y-11, Y-12 presentan resultados inferiores a los regulados en las normas objeto de estudio. Según la Norma Cubana (NC 827, 2017) y (NC 1021, 2014) las muestras de agua analizadas se encuentran por debajo del LMA por ellas $(0.05 \mathrm{mg} / \mathrm{L})$. 


\section{CONCLUSIONES}

- $\quad$ El $58.34 \%$ de las muestras estudiadas contienen níquel por encima de los LMA, el $8.33 \%$ se halla en el LMA y el $33.33 \%$ se localiza por debajo del LMA, según la Norma Cubana (NC 827, 2017) y (NC 1021, 2014). En el 66.66 \% de las muestras analizadas el Ni se halla por debajo del LMA, en el $25.00 \%$ se localizan por debajo del LMA y en el $8.34 \%$ se encuentra en el LMA por la norma de la (OMS, 2011a). En promedio, el níquel en las aguas del Río Yamanigüey es de $0.08 \mathrm{mg} / \mathrm{L}$.

- $\quad$ El $83.34 \%$ de las muestras analizadas contienen hierro por debajo de los LMA y el $16.66 \%$ se encuentran por encima de los LMA por las normas evaluadas. Como promedio, las 12 de las muestras estudiadas para analizar los contenidos de hierro se hallan por debajo de los LMA por las normas objeto de estudio.

- $\quad$ El $8.34 \%$ de las muestras estudiadas para analizar los contenidos de aluminio, se encuentran en el LMA y el $91.66 \%$ se encuentran por debajo del LMA por las normas estudiadas. Como promedio las 12 muestras estudiadas para analizar el aluminio en las aguas del Río Yamanigüey se encuentran por debajo del LMA.

- Según las normas cubanas el $100 \%$ de las muestras de aguas del Río Yamanigüey, contienen plomo por debajo del LMA. Al comparar el contenido de este elemento químico en el agua con la norma de la (OMS, 2011a), el $50 \%$ de las muestras analizadas se encuentra por encima del LMA y el $50 \%$ se encuentra por debajo del LMA.

- Según las normas evaluadas el $100 \%$ de las muestras estudiadas para analizar el cromo, manganeso, cobre y zinc se localizan por debajo de los LMA.

- El cobalto en las aguas del Río Yamanigüey oscila entre $0.00-0.03 \mathrm{mg} / \mathrm{L}$ en los 12 puntos muestreados.

- No hay presencia de cromo hexavalente en las aguas del Río Yamanigüey.

\section{REFERENCIAS}

APHA. (2016). Standard Methods for the Examination of Water and Wastewater 23rd Edition. Encyclopedia of Forensic Sciences: Second Edition, 522-527.

Arada Pérez, M. de los Á., Garrido Larramendi, D., \& Acebal Ibarra, A. T. (2018). Evaluación de metales pesados e impacto ambiental en los pozos "Rive Fuente" y "Bárbara" del poblado El Cobre. Revista Cubana de Química, 30(1), 68-76.

Argota-Pérez, G., \& Argota-Coello, H. (2012). Evaluación ambiental del río San Juan de Santiago de Cuba por exposición bioacumulativa a metales pesados. Medisan, 16(08), 1207-1214.
ATSDR. (2000). Bifenilos Policlorados Agencia para Sustancias Tóxicas y el Registro de Enfermedades.

Barea Pérez, I., Barrera, M., Alina, V., \& Infante, R. (2010). Estudio morfotectónico del área enmarcada por las concesiones mineras La Delta, Cantarrana y Santa Teresita. Minería y Geología, 26(1), 1-12.

Chávez-Vallarino, C. (2011). Detección de metales pesados en agua. Instituto Nacional de Astrofísica, Óptica y Electrónica, $51-54$.

Crespo-Lambert, M. (2018). EVALUACIÓN DE LA CALIDAD DE LAS AGUAS DE ABASTECIMIENTO DEL POBLADO YAMANIGÜEY. In Ingenieria geologa.

Domínguez-González, L., Rodríguez-Infante, A., Wobbe, F., Stanek, K. P., \& Gloaguen, R. (2007). Morfoalineamientos en la zona costera entre el poblado de Yamanigüey y la ciudad de Baracoa. Morfoalineamientos En La Zona Costera Entre El Poblado de Yamanigüey y La Ciudad de Baracoa, 23(3), 17.

Dunán Ávila, P. L. (2019). Evaluación de la calidad de las aguas superficiales del río Yamanigüey mediante el Índice de Calidad de Agua ICA-NSF. Universidad de Moa.

Espitia, N. (2015). Determinación de metales pesados en sedimentos superficiales en cuerpos de agua del canal del dique en las poblaciones de Gambote y Soplaviento (Bolívar). Revista Del Instituto de Investigación de La Facultad de Ingeniería Geológica, Minera, Metalurgica y Geográfica, 17(34).

Fernández Rodríguez, Moraima; Fernández Urgellés, O. (2007). Evaluación de la calidad físico-química y bacteriológica del agua subterránea en pozos criollos del municipio de Moa. Minería \& Geología, 23(4), 10.

Fernández-Rodríguez, M., Christian, B. N., Guardado-Lacaba, R., \& Carmenate, Y. A. (2018). Evaluación hidroquímica de las aguas del río Cayo Guam, Moa, Cuba. Minería y Geología. http://scielo.sld.cu/scielo.php?script=sci arttext\&pid=S1993-80122018000300003\&lng=es\&tlng= en.

Flores Lozano, H. H. (2016). Evaluación de la concentración de metales pesados en las aguas del rio grande y su relación con la actividad minera. Universidad Nacional de Cajamarca-UNC.

García-García, N., Pedraza-Garciga, J., Montalvo, J. F., Martínez, M., \& Leyva, J. (2012). EVALUACIÓN PRELIMINAR DE RIESGOS PARA LA SALUD HUMANA POR METALES PESADOS EN LAS BAHIAS DE BUENAVISTA Y SAN JUAN DE LOS REMEDIOS, VILLA CLARA, CUBA. Revista Cubana de Química, 24(2), 126-135.

Gonzáles V, Valle Sonia, Nirchio M., Olivero J., Tejeda L, Valdelamar J, P. F. \& G. K. (2018). Evaluación del riesgo de contaminación por metales pesados $(\mathrm{Hg}$ y $\mathrm{Pb})$ en sedimentos marinos del Estero Huaylá, Puerto Bolívar, Ecuador. Revista Del Instituto de Investigación de La Facultad de Ingeniería Geológica, Minera, Metalurgica y Geográfica, 21(41), 7582.

Lima Cazorla, L., Olivares-Rieumont, S., Columbie, I., De La Mederos, D. R., \& Gil Castillo, R. (2005). Niveles de plomo, zinc, cadmio y cobre en el rio Almendares, Ciudad Habana, 
Cuba. Revista Internacional de Contaminacion Ambiental, 21(3), 115-124.

Lopez Arisaca, S. R. (2018). Evaluación de la calidad de agua respecto a Metales Pesados presentes en el río Tambo provincia de Islay 2016 - 2018. Universidad Nacional de San Agustin, Arequipa.

NC 827. (2017). Agua potable - requisitos sanitarios. Oficina Nacional de Normalización (NC), Cuba, 261, 830-835.

NC 1021. (2014). Higiene comunal - fuentes de abastecimiento de agua - calidad y protección sanitaria. Oficina Nacional de Normalización (NC), Cuba, 261, 8-10.

OMS. (2011a). Guías para la calidad del agua de consumo humano. Organización Mundial de La Salud, 4, 608.

OMS. (2011b). Guías para la calidad del agua de consumo humano. Cuarta edición. Incorpora la primera adenda. Organización Mundial de La Salud, 4, 608.

Polanco-Almaguer, P. L. (2013). Influencia de los procesos geológicos en la morfología costera del municipio de Moa Ciencia \& Futuro, 3(2), 1-13.

Rivera, Hugo., Chira, Jorge., Chacón, Ítalo., Medina, André., \& Casallo, Irwin. (2013). Geodisponibilidad de metales pesados en sedimentos de los ríos Supe y Pativilca, departamento de Lima. Revista Del Instituto de Investigación de La Facultad de Minas, Metalurgia y Ciencias Geográficas, 13(26), 2125
Rubio, C., González Weller, D., Martín-Izquierdo, R. E., Revert, C., Rodríguez, I., \& Hardisson, A. (2007). El zinc: Oligoelemento esencial. In Nutricion Hospitalaria (Vol. 22, Issue 1, pp. 101-107). https://doi.org/10.23853/ bsehm.2001.0510

Salas Urviola, F. B. (2014). Determinación de metales pesados en las aguas del río Ananea debido a la actividad minera aurífera, Puno - Perú. Revista de Investigaciones, 5(4), 4753.

Vidal, N. F., Acosta, H. S., \& Villacres, F. G. (2018). Determinación de metales pesados en los cuerpos de agua del Área de Conservación Regional Humedales de Ventanilla, Región Callao, Perú. Revista Del Instituto de Investigación de La Facultad de Ingeniería Geológica, Minera, Metalurgica y Geográfica, 20(39), 149-158.

White, P. A., \& Rasmussen, J. B. (1998). The genotoxic hazards of domestic wastes in surface waters. Mutation Research Reviews in Mutation Research, 410(3), 223-236. https://doi. org/10.1016/S1383-5742(98)00002-7 